\title{
Anisotropia - Movements towards a Lyrical Design
}

\author{
Christoph Klemmt \\ Orproject \\ 21 Montpelier Street, London, SW7 1HF, UK \\ christoph@orproject.com
}

\begin{abstract}
As different as music and architecture are, the two forms of art also share a close relationship, and many composition and design concepts can become applicable to both. Historically the two disciplines have often been related on an emotional level, however the work of Orproject attempts a direct transformation from one to the other on an underlying structural level. By transforming time based attributes of music into spatial dimensions, different types of physical structures have been created which use lyrical effects to answer architectural requirements. The analysis of movement behaviours forms an intermediary step between the compositions in time and space.
\end{abstract}

\section{Architecture. Music. Algorithmic. Digital. Design. Composition. Flow.}

\section{ARCHITECTURE AND MUSIC}

Architecture and music are two very different forms of art, one is very long lasting while the other is very ephemeral. However there are also many relations between the two, and throughout history many parallels can be found. Music and sounds as well as spaces and buildings can sometimes evoke very similar emotions, and many of the words we use to describe both disciplines are the same, such as 'composition', 'structure' or 'rhythm'.

Although music and architecture were traditionally treated quite separately, their relations started to be philosophically investigated more closely especially during Romanticism, for example by the Schlegel brothers or by Wilhelm Joseph Schelling. The latter appears to have coined the term of architecture being 'frozen music', a metaphor which has been famously quoted by Johann Wolfgang von Goethe. (Pascha 2004)

\subsection{Program music}

Direct translations of one discipline to the other started to appear in classical music with program music. In program music, opposed to absolute music, the composers attempt to musically describe subjects which are outside of the realm of music itself, such as stories, situations or spaces. Early program music can be found in the Renaissance and Classical periods, but it started to be embraced especially during Romanticism, and composers often described spaces and landscapes, and sometimes specifically buildings and architectural spaces. An example for this is the cycle Pictures at an Exhibition by Modest Mussorgsky.

\subsection{Pictures at an exhibition}

In the cycle he musically describes the visit through an exhibition of paintings by the Russian artist and architect Viktor Hartmann, which was organised by his friends after his unexpected death. Mussorgsky describes 10 of the paintings in the different movements, and he also describes the space and the walk from one picture to the next through the 'Promenades' between. (Russ 1992, Struck 1983)

The most famous of the movements is titled The Bogatyr Gates, which depicts Hartmann's design sketch for new city gates in Kiev. By narrating the drawing through his composition, Mussorgsky musically describes a building, or rather the un-built proposal for a building, he has turned architecture into music.

This transformation happens on two levels: Firstly on an emotional level. The impressive city gates are translated into an impressive music, the strong standing building is described through powerful and strong chords. Mussorgsky catches the atmosphere of the building and recreates it with the composition, as in film music, the emotions of a situation are translated and emphasised.

On the second level, Mussorgsky creates sounds with his composition which are similar to the sounds which we would expect to hear around the 
building. Those include the sound of the bells which are visible in the tower of the gates, but also the regular metric rhythm of the composition reflects the regular movement of the visitor on his approach towards the building.

\subsection{Notation}

Both music and architecture are forms of art which require additional mediums for their transmission and proliferation, other than just the piece itself or a depiction of it: Both are transmitted via a notation, via drawings which are made to describe the sounds or a building to other people, who are then able to create or re-create the intended piece. In musical notation this happens via a symbolisation, in architecture via a combination of both diagrammatic depiction and symbolisation. (ShawMiller 2006)

\subsection{Script}

Already the notation of script takes sound and turns it into a physical product, into a formation of ink on paper, or into carved grooves within a base material. The first step was the development of a symbol: The material formations or abrasions symbolise meanings which are orally represented by sounds, and their notation becomes a physical manifestation of those sounds in a quasi twodimensional object.

\subsection{Time and space}

Importantly, in a second step the symbols have been placed in an order and they are read in a certain direction. With all writings we start to read at a start point, and following the symbols we move towards the end, usually in a linear manner, or quite often in a broken linear manner as in a text which is written across several rows and pages.

Like this, time has been translated into space. The spoken sentence unfolds for a specific duration in time, the written symbolisation of it unfolds on the paper in space, the time dimension has become a spatial dimension.

\subsection{Musical notation}

Similarly in musical notation, the time dimension gets transformed into a linear or broken linear spatial dimension. One of the most important aspects of music which gets symbolised in the notation is the pitch, the base frequency of the sounds which are to be created. In most musical notations, the notation of the pitch happens in a second dimension: Where the time dimension is read in a horizontal manner, the pitch is read vertically. This applies to the notation using modern musical symbols, but it also applies to early Western or Asian forms of musical notation.
Also the pitch of a sound is a property which is time-based, it is the duration at which the waveform of a sound is repeated in time. Therefore also the notation of the pitch in the vertical direction is a transformation of time to space.

In gramophone records and CDs, both time and frequency of music are transformed into physical objects, in both cases the time dimension gets transformed into a curved linear space dimension.

\section{COMPOSITION IN SPACE AND TIME}

Orproject attempted to use this transformation from time to space in order to generate a music-based architecture. In a first step, a musical composition logic has been developed, which was used for Klavierstück I, a composition for piano by Orproject director Christoph Klemmt. The compositional structure uses the modulations of a twelve tone row in order to generate constantly evolving rhythmic structures using effects similar to sub-Saharan Baganda music, which have also been used by György Ligeti in his Études pour Piano. (Dibelius 1994)

\subsection{Baganda music}

Baganda music is the traditional music of the Ganda people of Uganda, but similar rhythmic structures are also appearing in traditional music in other parts of Africa. One of the main instruments used is the Amadinda, a type of Xylophone with 12 tones tuned in equi-pentatonic scale. It is played by three musicians, one of which sits opposite the other two in front of the instrument, and it is played at a constant, fast meter.

The first musician starts by playing a repetitive pattern in parallel octaves, which may have a length of usually 12,18 or 24 beats. The second musician then starts to play a pattern of the same length, always placing his beats between the metric beats of the first player, resulting in a combined cycle of 24 beats, at twice the speed of the individual cycles.

Audible are not anymore the individual patterns of the two musicians, but a combination of the two. Due to the fast speed they are perceived not as a melody, but as a rhythm of the highest or lowest tones which stand out above the basic meter.

The third musician then starts to play the intrametric pattern which is resulting from the first two players on the lowest keys, but transposed up by two octaves. By emphasizing certain beats, the musicians can then bring different rhythmic patterns to the foreground. (Kubik 1983) 


\subsection{Structural composition logic}

Using this effect, Klavierstück $\mathrm{I}$ is based on a simple component, a short musical theme, which extends across both a time and frequency range. The motif is a twelve tone row, spanning the range of an octave. It is executed at a repetitive, fast metric, consequently resulting in a duration of 12 tones.

This basic component of the composition is being played on the piano by both hands simultaneously, each voice executing its row separately, but alternating between tones in the same way as the first two players of the Amadinda do. However the two rows are shifted against each other by about a third of their length, therefore resulting in 24 tones which are played at the duple meter of the individual twelve tone row. Like this the twelve tone row is not being used as a melodic order as in Schönberg's Dodecaphony, but as in the Baganda music only the highest tones of the two rows become audible in the form of a rhythmic pattern.

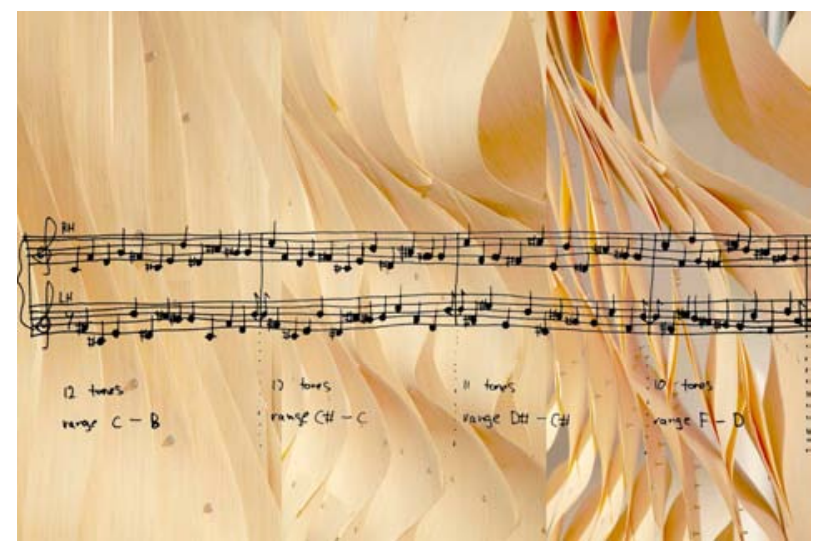

Figure 1: Klavierstück I: Structural aggregations

The components in the two voices are now being repeated, forming an aggregation in the time dimension, and forming the basic, so far regular, structural system of the composition. However, different from the Baganda music, a morphological shift happens with each repetition of the component, the twelve tone row gets transformed: Regarded as an order of tones within a range rather than as a melody, this range of its execution can be shifted. By shifting the range up by a half tone, all the tones in the row remain in their position except for the lowest tone. The previously lowest tone still remains its position within the row, but it gets transposed up by an octave. As a result the twelve tone row remains the same, but its range has been shifted, and as a consequence also a different but similar set form the audible upper tones within the row, which generate a different but similar rhythmic pattern.
The range of execution of the twelve tone row can not only be shifted up or down in this way, but it can also be reduced in its size. In this case those tones will drop out of the row which are outside of the range, and the next tone in the order is played instead. By keeping the metric structure, the reduction of the range then also results in a reduction of the length of the cycle, the basic musical component gets transformed across both frequency and time. The components can also be shifted differently within the two voices, both in the frequency and in the time directions.

This now forms an irregular structural system for the composition, which has been used in Klavierstück I to create a continuously developing rhythmic morphology, leading from the lowest key of the piano to the highest. Perceived is not a melody, but a floating field of tones and the continuously evolving rhythms.

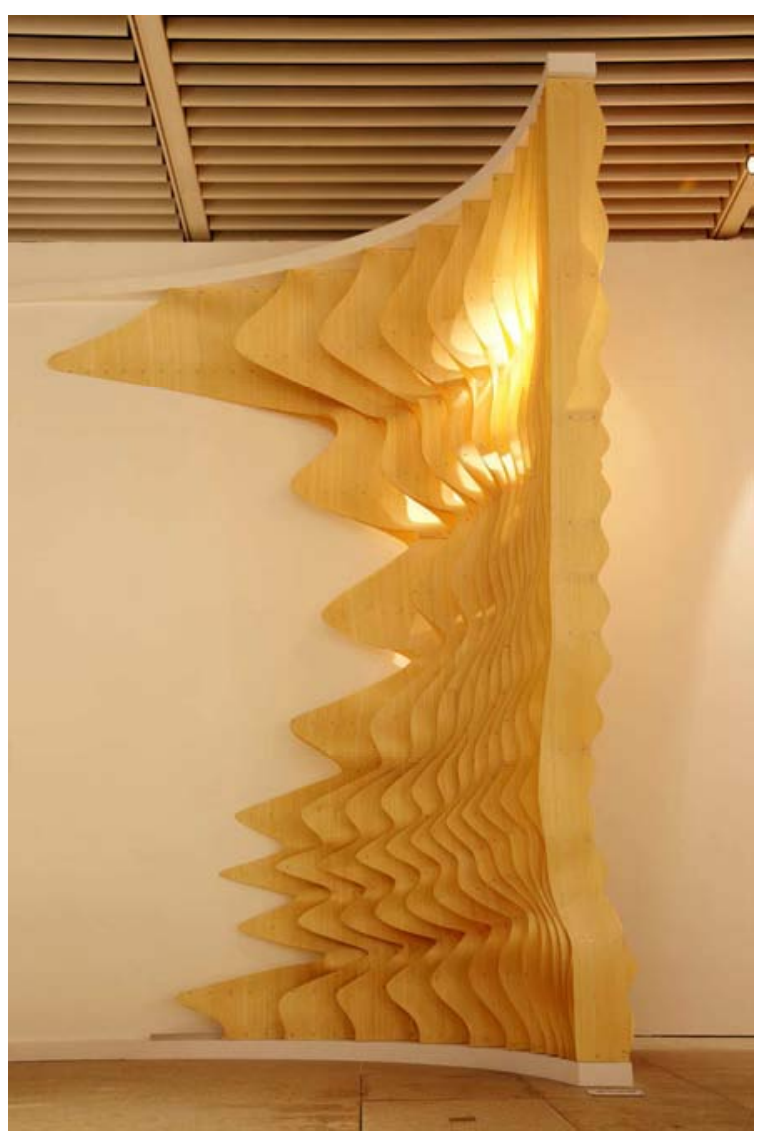

Figure 2: Anisotropia: Lyrical wall system

\subsection{Anisotropia}

In a similar way to Klavierstück I, an architectural structural system was set up for Anisotropia, which becomes the physical manifestation of the composition, a frozen piece of music. The installation, designed by Xin Wang and Christoph Klemmt of Orproject, is also based on a simple, this time spatial component: An element of two connected lamella of different length. Due to its 
connections the basic component unfolds in three dimensional space, the strips create an opening and form an extrusion in the third dimension.

The component is repeated in space rather than in time, within the two directions defined by its opening. This aggregation results in a wall system, the component creates the structure, openings and rhythm within itself. However, as in the piano composition, the component is modified with each iteration: A scaling of its height and width can create different densities and permeabilities, and a modification of the internal and external extrusion can control the depth behaviour of the structure.

\subsection{Structure and light}

This allows for a complex control of the view, light and shading properties of the wall system. In Klavierstück I, the underlying structure was used to create a musical effect, the rhythmic transformations. In Anisotropia, the system is used to respond to architectural requirements instead.

It has been tested as an installation at the China National Museum in Beijing. Layers of bamboo lamella form the wall system and control the visitor's experience across the installation. In the piano piece only ever the upper few tones of each twelve tone row are audible and create the floating field of rhythmic transformations. Similarly in the installation, only the peaks of each strip become visible and create a floating field of structure, shadow and light.

\subsection{Curved non-linearity}

The notation of music along straight lines is useful, and Anisotropia is the geometric prototype for a façade system, tested along the short length of a straight line which forms the transformation of the time direction into space. However for the creation of architecture, time can be translated into space in a wide range of other possibilities.

Conceptually, between a time based system such as sound or music, and a space based system such as three dimensional form, a third type of system can be defined which relies on both time and space: the systems of moving objects. The movement of an object always happens within both space and time, and an analysis of the abstracted movement of objects past each other and past deflectors was used as a tool to control the way in which the time dimension can be translated into space.

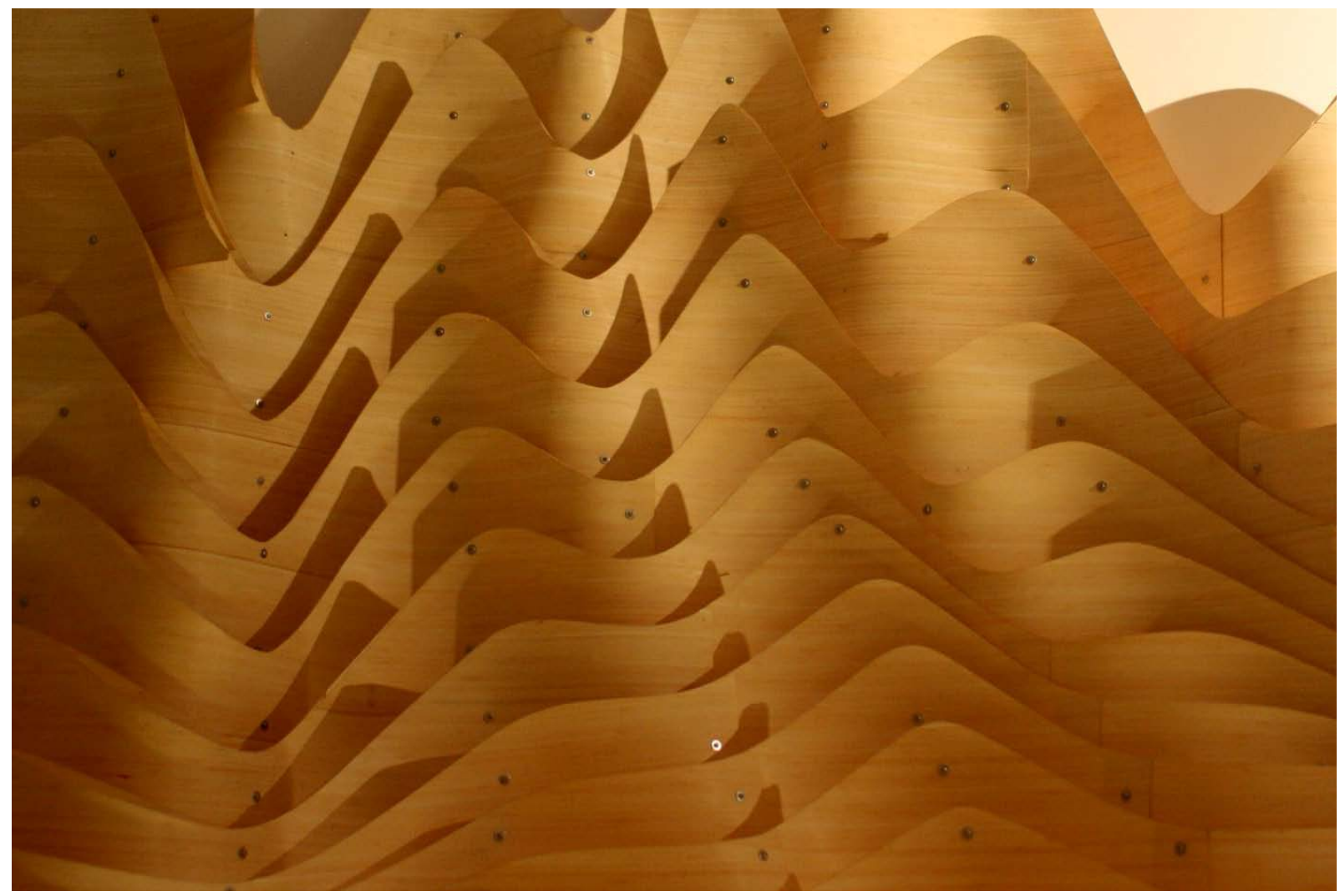

Figure 3: Anisotropia: Light and shadow 
Orproject therefore investigated algorithms to describe time-based flow patterns of particles, and translating those into permanent spatial configurations via their trajectories. The particle movement evolves in both space and time dimensions, their trajectories become the possible spatial directions for the translation of the time dimension of music into space.

\subsection{Flow}

Particular interest was given to abstracted flow simulations past deflectors, similar to planets moving around each other, or similar to the flow of particles past electrodes. The behaviours are influenced similarly to interparticular forces, the strength of which depends on the distance between influencing units, and it is calculated iteratively via their vector behaviour across a field.

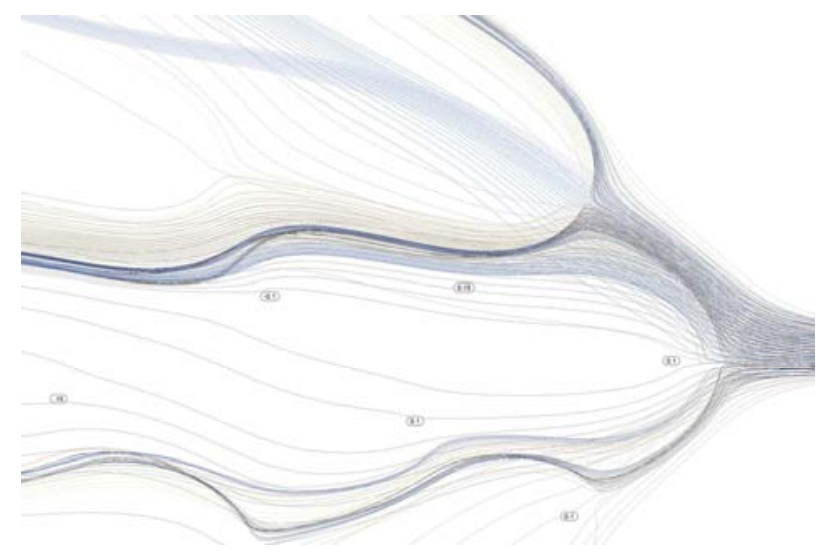

Figure 4: Flow: Between space and time

Different types of forces and different types of cross-unit influences have been used in the simulations. A base velocity is a linear velocity of the request object in one direction. Independent of other acting forces, the base velocity, if applied, will remain a constant addend.

The start velocity is a movement vector of the request object at the beginning of the simulation, or at its point of entry into the system. The start velocity can change during the consecutive iterations, whereas the base velocity will remain the same.

A base factor can be used to constantly influence the movement of the request object, by multiplying the movement vector with it in each iteration. If set to a value between 0 and 1 , the speed of the object will be decelerated similar to a movement influenced by air drag.

The non-linear forces are those which depend on the distance of the request object to the deflector. A deflector can either attract or repulse the object, and the deflector can be either static or mobile itself. The distance between the request object and the deflector can be an addend to the velocity, or it can be an influencing factor onto it. The following types of behaviours have been used to calculate the influences of the deflectors:

A hyperbolic influence, where 1/distance describes the influence of the deflector onto the object. This behaviour is similar to the way gravitational forces act between objects.

However as objects or particles don't move within a certain distance from each other, the hyperbolic influence can be refined by a counter influence of $1 /$ distance $^{2}$, which creates an opposing influence for small distances.

Both of those influences will act on the objects at every point even for large distances, which can cause difficulties for a detailed control over the trajectories. Therefore a cut-off distance can be set, or the influence can be calculated using a cosinal behaviour for distances within a defined range around the deflector.

The benefit of the simulations lie in the possibility to calculate flows past pre-existing conditions, they become applicable information for the development of architectural space defined by site conditions and architectural requirements.

\subsection{Kinetika}

The basic movement of a request object can happen in three ways: The movement can originate externally and be assumed linear at the point of entry of the simulation, as it would be the case with particles moving past electrodes. The movement can originate from the deflectors, as in the case of simulations of magnetic fields. Or the movement can be orthogonal to the direction towards the deflectors, as in the movement of planets around a star.

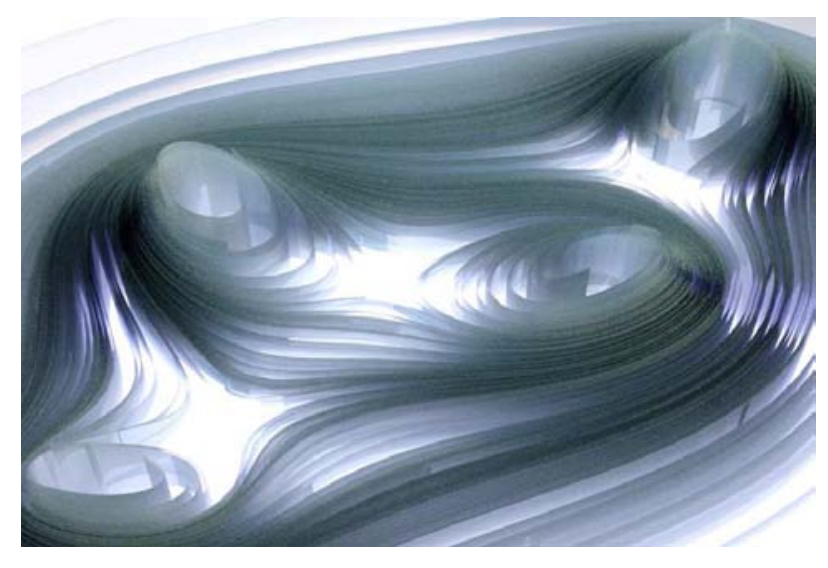

Figure 5: Kinetika: Attractor-orthogonal flow simulation 
This last type of movement has been visualised in Orproject's Kinetika, which shows a twodimensional movement around deflectors. The third dimension reflects the velocity of the request point during its movement.

\subsection{Deflection of directional flows}

On the contrary to the deflector-orthogonal flows, the directional flows need either a base or a start velocity for their calculation. The flow of the object then gets distorted by the deflectors, which can act as either attractors or as repulsors. For architectural applications, the deflectors can be used to guide the request objects across a site, so that the deflectors can be positioned according to existing site conditions or to programmatic requirements, or they can be placed to influence the behaviour of the trajectories in specific ways.

\subsection{Busan opera house}

This possibility has been utilised for the design proposal for Busan Opera House, in which the trajectories resulting from a flow simulation have been applied to guide the facades of the building. The wall system of Anisotropia has been applied in multiple layers, with its time direction translated along the non-linear flow patterns.

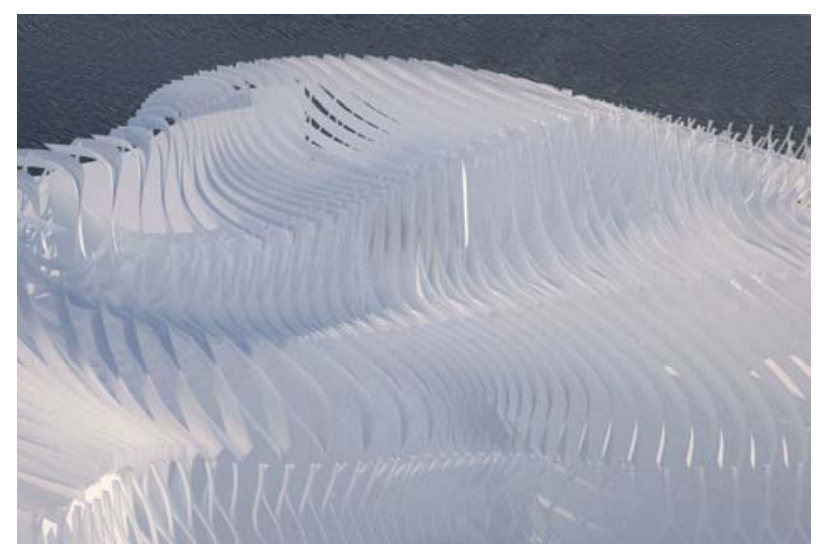

Figure 6: Busan Opera House: A lyrical structure

The distribution of the programmatic elements on the site is used as the deflector set that guides the flow of the trajectories, each deflector acts according to the magnitude of its attraction and the range of its influence. The different elements of the building, such as the atrium, the two auditoria and the back of house spaces, have been assigned attraction and repulsion attributes depending on their requirements, so that the resulting flow pattern of the simulation is enclosing the spaces around them, forming the base lines for the facades of the building.

The site is situated on an artificial island between the sea and the city. The flow of the walls has been designed as seven separate layers, originating from the sea and moving towards the city. Along their way the trajectories flow around the building elements such as the theatre and auditoriums, splitting up and being diverted by the deflectors.

\subsection{Structure and light}

As in the installation Anisotropia, the proposed façade structure becomes a physical manifestation of Klavierstück I. For Busan Opera House, the strip morphology is constructed from curved steel sections. The seven parallel layers form the façade and their alteration results in a complex behaviour of the architectural rhythms, which are used to control the light, shading and view properties across the façade.

Towards the sea side of the building, at the start of the flow simulation, the layers are aligned and appear as a single structure. However slowly the flows of the separate trajectories begin to shift against each other, and the alignment of the façade components starts to break apart. A complex field of varied façade rhythms begins to emerge.

The façade structure is altered in the length of its repetition, but also the orientation and the depth of the extrusions are manipulated in order to control the view and light, depending on the programmatic requirements on the inside of the building. Around spaces such as the atrium or restaurants, the façade is open and translucent, allowing light to come in and allowing views onto the sea. In front of the auditoria and rehearsal rooms, the façade becomes dense and closed, focussing the attention of the users onto the inside of the building.

\subsection{Music and architecture}

As different as music and architecture are, the two forms of art also have very close relations, and many composition and design concepts can become applicable to both. However the importance lies in finding and using the relevance of their relation, so that both music and architecture can benefit from each other. 


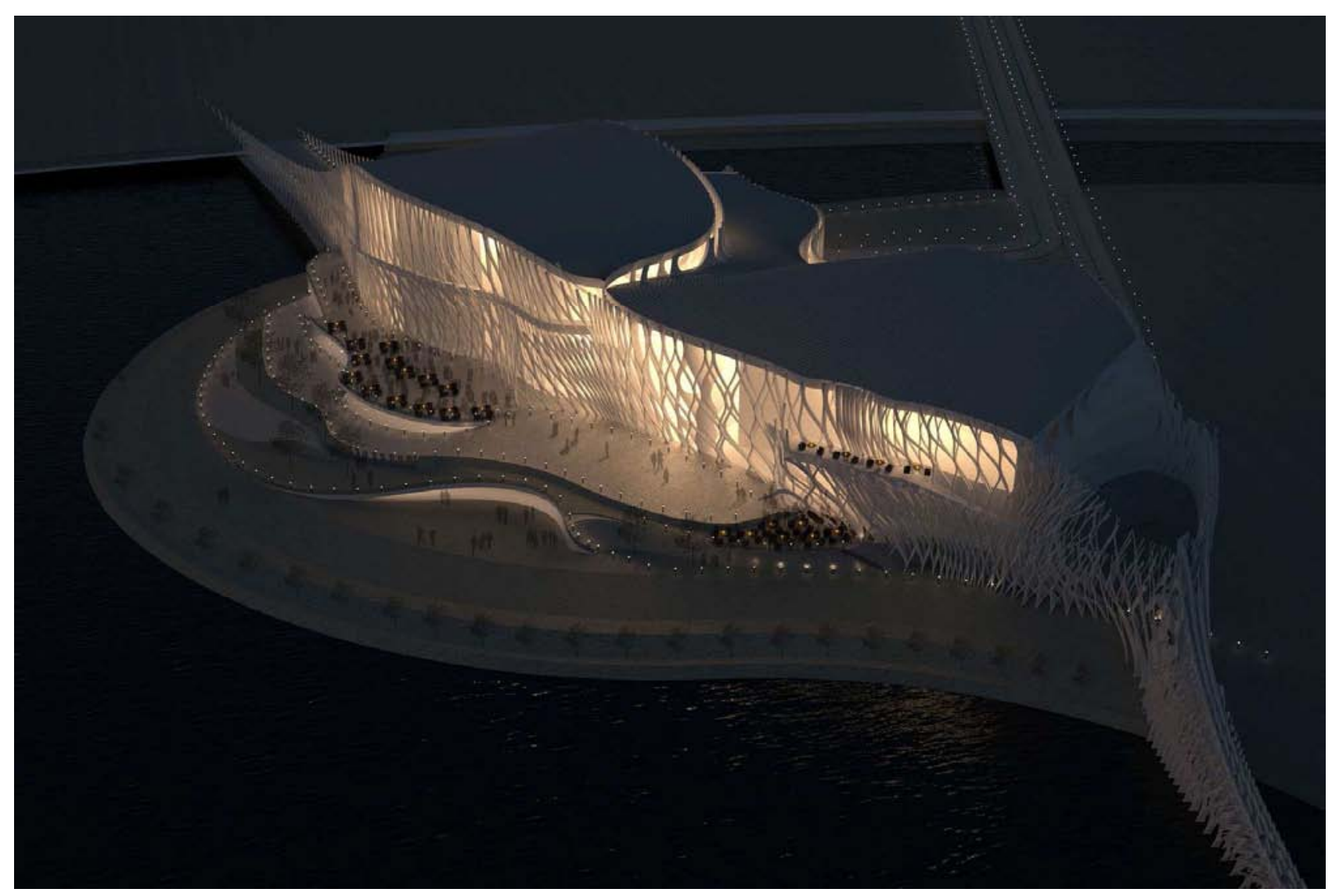

Figure 7: Busan Opera House: Light and shadow

\section{BIBLIOGRAPHY}

Schelling, F. W. J. (1976) Philosophie der Kunst. Darmstadt

Pascha, K. S. (2004) 'Gefrorene Musik' - Das Verhältnis von Architektur und Musik in der ästhetischen Theorie. Berlin

Russ, M. (1992) Musorgsky: Pictures at an Exhibition. Cambridge Music Handbooks. Cambridge and New York: Cambridge University Press

Struck, M. (1983) Bild im Wort - Anmerkungen zu Mussorgskijs 'Bilder einer Ausstellung' und ihrem Programm. in Hamburger Jahrbuch für Musikwissenschaft BdG, Programmusik - Studien zu Begriff und Geschichte einer umstrittenen Gattung, Laaberverlag, Hamburg
Shaw-Miller, S. (2006) Thinking Through Construction: Notation - Composition - Event, The Architecture of Music. AA Files, No. 53, pp. 38-47

Dibelius, U. (1994) György Ligeti - Eine Monographie in Essays. B. Schott's Söhne, Mainz

Kubik, G. (1983) Die Amadinda-Musik von Buganda. Musik in Afrika. Hrsg. Arthur Simon, (Staatliche Museen) Berlin, pp. 139-165

Lorenz, E. N. (1963) Deterministic Nonperiodic Flow. J. Atmos. Sci., 20, pp. 130-141

Martin, E. (ed) (1994) Pamphlet Architecture 16 Architecture as a Translation of Music. Princeton Architectural Press, New York 